Wilfrid Laurier University

Scholars Commons @ Laurier

Physics and Computer Science Faculty

Publications

Physics and Computer Science

$12-1990$

\title{
Polarization Characteristics of Double-Clad Elliptical Fibers
}

\author{
Feng Zhang \\ University of Waterloo \\ John W.Y. Lit \\ Wilfrid Laurier University, jlit@wlu.ca
}

Follow this and additional works at: https://scholars.wlu.ca/phys_faculty

\section{Recommended Citation}

Zhang, Feng and Lit, John W.Y., "Polarization Characteristics of Double-Clad Elliptical Fibers" (1990).

Physics and Computer Science Faculty Publications. 16.

https://scholars.wlu.ca/phys_faculty/16

This Article is brought to you for free and open access by the Physics and Computer Science at Scholars Commons @ Laurier. It has been accepted for inclusion in Physics and Computer Science Faculty Publications by an authorized administrator of Scholars Commons @ Laurier. For more information, please contact scholarscommons@wlu.ca. 


\title{
Polarization characteristics of double-clad elliptical fibers
}

\author{
Feng Zhang and John W. Y. Lit
}

\begin{abstract}
A scalar variational analysis based on a Gaussian approximation of the fundamental mode of a double-clad elliptical fiber with a depressed inner cladding is studied. The polarization properties and graphic results are presented; they are given in terms of three parameters: the ratio of the major axis to the minor axis of the core, the ratio of the inner cladding major axis to the core major axis, and the difference between the core index and the inner cladding index. The variations of both the spot size and the field intensity with core ellipticity are examined. It is shown that high birefringence and dispersion-free orthogonal polarization modes can be obtained within the single-mode region and that the field intensity distribution may be more confined to the fiber center than in a single-clad elliptical fiber.
\end{abstract}

\section{Introduction}

Recent progress in fiber technology by using phase or polarization modulation has given rise to new systems not only in optical communications but also in fiber sensors and in integrated optics. The ability to control and to maintain the polarization states is important in many systems, such as coherent communication systems and interferometric and polarimetric fiber sensors. The key to acquire such ability is to use high performance single-mode polarization-maintaining fibers and components. A highly birefringent fiber, in which the polarization state of the input light is preserved by minimizing the coupling between the two orthogonally polarized fundamental modes, is one of the most popular polarization-maintaining fibers. Such a fiber can be made by introducing transverse geometric asymmetry to the fiber core ${ }^{1-6}$ or by building in transverse asymmetric stresses in the region surrounding the core. ${ }^{7-10}$ Stress-induced birefringent fibers require manufacturing trade-offs between polarization holding and attenuation. ${ }^{11}$ The most serious problem with these fibers, however, is that the stresses induced by different thermal expansions make polarization holding sensitive to temperature variations and

The authors are with University of Waterloo, Physics Department, Guelph-Waterloo Program for Graduate Work in Physics, Waterloo, Ontario N2L 3G1, Canada.

Received 20 October 1989.

0003-6935/90/365336-07\$02.00/0.

(C) 1990 Optical Society of America. restrict their practical uses. ${ }^{12-14}$ In addition, accessing the guiding region of the fiber is a complex operation which changes the internal stresses; this adversely affects the use of the fiber to make polarization-maintaining or polarization-controlling components, such as couplers, polarizers, etc. On the other hand, if birefringence is caused by fiber geometry, bending and temperature could change over wide ranges without significantly degrading the optical performance. Such a fiber also allows easy access to the guiding region, which could be achieved by various methods such as etching and polishing, without adversely affecting the performance. Among fibers of this type, single-clad elliptical (SCE) fiber has emerged as the most practical one. It has been studied both theoretically $1,2,15,16$ and experimentally. ${ }^{4}$ The birefringence of such a fiber can be increased by increasing the ellipticity of the core or the index difference between the core and the cladding. To produce high birefringence, both the core ellipticity and the index difference have to be large, resulting in the core size being very small for a single-mode fiber. ${ }^{2}$ This does not only make fabrication and connection more difficult, but it also enhances the nonlinear effects in the core.

To overcome these disadvantages, depressed inner cladding elliptical (DICE) fibers with self-locating Dshape have been fabricated. ${ }^{17}$ The polarization-maintaining characteristics of DICE fibers have been investigated experimentally. ${ }^{18}$ Theoretically they have been analyzed by means of a double-clad rectangular waveguide (DCRW) model. ${ }^{19}$ The results show that DICE fibers, compared with SCE fibers, have higher birefringence in the weakly guiding region, zero polarization-mode dispersion in the single-mode region, and larger first-higher-mode cutoff frequency, leading to a larger core size for single-mode operation. Both the 
beat length and the cutoff wavelength can be predicted reasonably well. ${ }^{18}$ But the model cannot give the mode field distribution and spot size, which are essential to the proper design of experiments, connections, and fabrication of components.

In this paper we use the Gaussian approximation to calculate the spot size, the field distribution, the birefringence, and the polarization-mode dispersion in weakly guiding DICE fibers. We examine the effects of a depressed inner cladding and core ellipticity on these quantities.

\section{Gaussian Approximation for DICE Fibers}

We studied the structure shown in Fig. 1. A DICE fiber has the following parameters: $a_{1}$ and $a_{2}$ are the major axes of the core and of the inner cladding; $b_{1}$ and $b_{2}$ are the minor axes of the core and of the inner cladding; $n_{0}, n_{1}$, and $n_{2}$ are the refractive indices of the core, inner and outer cladding respectively. In terms of elliptical coordinates $\xi$ and $\eta$, the two boundaries of the inner cladding may be expressed by $\xi=\xi_{1}$ and $\xi=$ $\xi_{2}$.

The refractive index distribution in a DICE fiber can be written as (see Fig. 1)

$$
n^{2}(\xi)=n_{0}^{2}\left[1-2 \Delta_{2} f(\xi)\right]
$$

where

$$
\begin{aligned}
& f(\xi)=\left\{\begin{array}{cc}
0 & \xi<\xi_{1}, \\
\alpha H\left(\xi-\xi_{1}\right), & \xi_{1} \leq \xi<\xi_{2}, \\
H\left(\xi-\xi_{2}\right), & \xi \geq \xi_{2},
\end{array}\right. \\
& H(x)= \begin{cases}0, & x<0, \\
1, & x \geq 0,\end{cases} \\
& \alpha=\frac{\Delta_{1}}{\Delta_{2}}=\frac{n_{0}^{2}-n_{1}^{2}}{n_{0}^{2}-n_{2}^{2}},
\end{aligned}
$$

with

$$
\Delta_{1}=\frac{n_{0}^{2}-n_{1}^{2}}{2 n_{0}^{2}}, \quad \Delta_{2}=\frac{n_{0}^{2}-n_{2}^{2}}{2 n_{0}^{2}} .
$$

We also introduce the parameters

$$
R_{x}=\frac{a_{2}}{a_{1}}, R_{y}=\frac{b_{2}}{b_{1}} \text {. }
$$

If $\Delta_{1}=0$ or $=\Delta_{2}$ and $R_{x}=R_{y}=1$, a double-clad elliptical fiber becomes a single-clad circular fiber. The normalized frequencies can be defined as

$$
\begin{aligned}
& V_{x}=k a_{1} n_{0}\left(2 \Delta_{2}\right)^{1 / 2}, \\
& V_{y}=k b_{1} n_{0}\left(2 \Delta_{2}\right)^{1 / 2} .
\end{aligned}
$$

The fundamental modes of a weakly guiding elliptical fiber are plane polarized parallel to the major and minor axes of the ellipse. The transverse fields have the forms

$$
\begin{aligned}
& E_{x}=\Psi(x, y) \exp \left[i\left(\beta+\delta \beta_{x}\right) z\right], \\
& E_{y}=\Psi(x, y) \exp \left[i\left(\beta+\delta \beta_{y}\right) z\right] .
\end{aligned}
$$

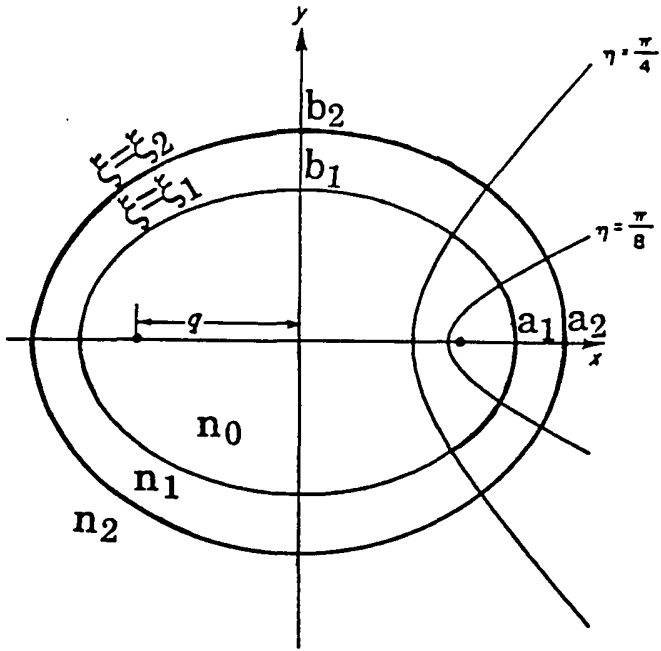

(a)

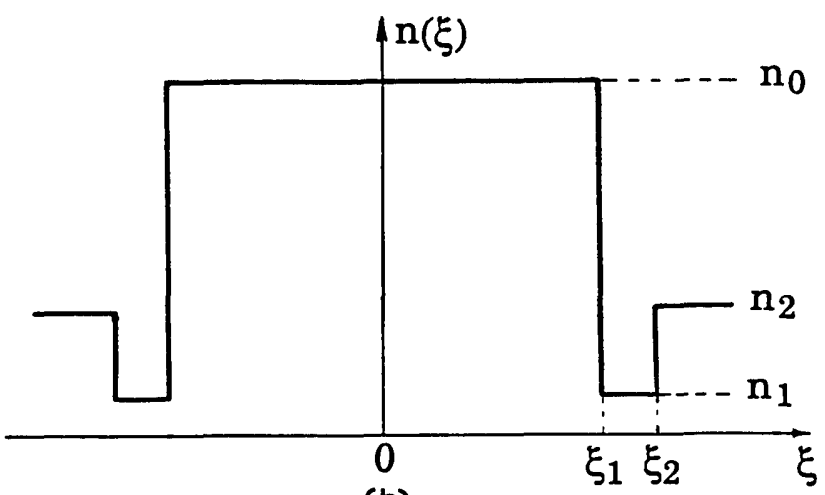

(b)

Fig. 1. (a) Schematic diagram of a double-clad elliptical fiber with depressed inner cladding. (b) Refractive index distribution in the radial direction.

Parameter $\Psi(x, y)$ is the scalar wave equation solution which has the scalar propagation constant $\beta$, i.e.,

$$
\left[\nabla_{t}^{2}+k^{2} n^{2}(x, y)-\beta^{2}\right] \Psi(x, y)=0,
$$

where $k$ is the wave number in vacuum and $\nabla_{t}$ is the transverse gradient operator. Parameters $\delta \beta_{x}$ and $\delta \beta_{y}$ are the polarization corrections resulting from the waveguide structure, which are given by ${ }^{20}$

$$
\delta \beta_{p}=\frac{\rho_{p}\left(2 \Delta_{2}\right)^{3 / 2}}{2 V_{p}} \frac{\int_{-\infty}^{\infty} \int_{-\infty}^{\infty}\left(\nabla_{\mathfrak{t}} \cdot \mathbf{e}_{\mathfrak{t}}\right) \mathbf{e}_{\mathfrak{t}} \cdot \nabla_{t} f(x, y) d x d y}{\int_{-\infty}^{\infty} \int_{-\infty}^{\infty} \mathbf{e}_{\mathfrak{t}}^{2} d x d y}, p=x, y,(10)
$$

with $\rho_{x}=a_{1}, \rho_{y}=b_{1}$, and $\mathbf{e}_{\mathbf{t}}=E_{x} \mathbf{i}+E_{y} \mathbf{j}$. As in the case of a SCE fiber, we approximate the fundamental mode of a DICE fiber by a 2-D Gaussian function ${ }^{16,20 \text { : }}$

$$
\Psi(x, y)=\exp \left[-\frac{1}{2}\left(\frac{X^{2}}{W_{x}^{2}}+\frac{Y^{2}}{W_{y}^{2}}\right)\right],
$$

where $X=x / a_{1}$ and $Y=y / b_{1}$ and $W_{x}, W_{y}$ are the normalized spot sizes, ${ }^{21}$ which are the variational parameters to be determined along the major and minor 
axes. The variational expression for the propagation constant $\beta$ given by Eq. (9) can be written as ${ }^{20}$

$$
\beta^{2}=\frac{\int_{-\infty}^{\infty} \int_{-\infty}^{\infty}\left[k^{2} n^{2}(X, Y) \Psi-\left(\frac{\partial \Psi}{\partial X}\right)^{2}-\left(\frac{\partial \Psi}{\partial Y}\right)^{2}\right] d X d Y}{\int_{-\infty}^{\infty} \int_{-\infty}^{\infty} \Psi^{2} d X d Y}
$$

The extremum equations,

$$
\frac{\partial \beta}{\partial W_{x}}=0, \quad \frac{\partial \beta}{\partial W_{y}}=0,
$$

lead to the spot size equations, ${ }^{20}$

$$
\begin{aligned}
& \frac{\pi}{V_{x}^{2}}=\frac{W_{x}}{W_{y}} \int_{-\infty}^{\infty} \int_{-\infty}^{\infty} X \frac{\partial f}{\partial X} \exp \left(-\frac{X^{2}}{W_{x}^{2}}-\frac{Y^{2}}{W_{y}^{2}}\right) d X d Y, \\
& \frac{\pi}{V_{y}^{2}}=\frac{W_{y}}{W_{x}} \int_{-\infty}^{\infty} \int_{-\infty}^{\infty} Y \frac{\partial f}{\partial Y} \exp \left(-\frac{X^{2}}{W_{x}^{2}}-\frac{Y^{2}}{W_{y}^{2}}\right) d X d Y .
\end{aligned}
$$

Changing the coordinates $X$ and $Y$ in Eqs. (14) and (15) to elliptical coordinates $\xi$ and $\eta$,

$$
\begin{aligned}
& X=Q_{x} \cosh \xi \cos \eta, \\
& Y=Q_{y} \sinh \xi \sin \eta,
\end{aligned}
$$

with $Q_{x}=q / a_{1}$ and $Q_{y}=q / b_{1}$, we may obtain the spot size equations in the elliptical coordinates,

$$
\begin{aligned}
& \frac{W_{y}}{W_{x} V_{x}^{2}}+\frac{W_{x}}{W_{y} V_{y}^{2}}=2 \int_{0}^{\infty} Q_{x} \cosh \xi Q_{y} \sinh \xi \frac{\partial f}{\partial \xi} \exp [-A(\xi)] I_{0}[B(\xi)] d \xi \\
& \frac{W_{y}}{W_{x} V_{x}^{2}}-\frac{W_{x}}{W_{y} V_{y}^{2}}=2 \int_{0}^{\infty} Q_{x} \cosh \xi Q_{y} \sinh \xi \frac{\partial f}{\partial \xi} \exp [-A(\xi)] I_{1}[B(\xi)] d \xi
\end{aligned}
$$

where $I_{0}(x)$ and $I_{1}(x)$ are modified Bessel functions of the first kind and of orders 0 and 1 , respectively, and

$$
\begin{aligned}
& A(\xi)=\frac{W_{x}^{2} Q_{y}^{2} \sinh ^{2} \xi+W_{y}^{2} Q_{x}^{2} \cosh ^{2} \xi}{2 W_{x}^{2} W_{y}^{2}}, \\
& B(\xi)=\frac{W_{x}^{2} Q_{y}^{2} \sinh ^{2} \xi-W_{y}^{2} Q_{x}^{2} \cosh ^{2} \xi}{2 W_{x}^{2} W_{y}^{2}} .
\end{aligned}
$$

These equations can be used for a weakly guiding elliptical fiber with any refractive index profile. For a double-clad elliptical step-index fiber, $f$ is given by Eq. (2), so that substituting

$$
\frac{\partial f}{\partial \xi}=\alpha \delta\left(\xi-\xi_{1}\right)+(1-\alpha) \delta\left(\xi-\xi_{2}\right)
$$

into Eqs. (18) and (19) the spot size equations of DICE fibers can be finally obtained

$$
\begin{aligned}
& \frac{W_{y}}{W_{x} V_{x}^{2}}+\frac{W_{x}}{W_{y} V_{y}^{2}}=2\left\{\alpha \exp \left[-A\left(\xi_{1}\right)\right] I_{0}\left[B\left(\xi_{1}\right)\right]\right.\left.+(1-\alpha) R_{x} R_{y} \exp \left[-A\left(\xi_{2}\right)\right] I_{0}\left[B\left(\xi_{2}\right)\right]\right\}, \\
& \frac{W_{y}}{W_{x} V_{x}^{2}}-\frac{W_{x}}{W_{y} V_{y}^{2}}=2\left\{\alpha \exp \left[-A\left(\xi_{1}\right)\right] I_{1}\left[B\left(\xi_{1}\right)\right]\right. \\
&\left.+(1-\alpha) R_{x} R_{y} \exp \left[-A\left(\xi_{2}\right)\right] I_{1}\left[B\left(\xi_{2}\right)\right]\right\},
\end{aligned}
$$

where

$$
\begin{aligned}
& A\left(\xi_{1}\right)=\frac{W_{x}^{2}+W_{y}^{2}}{2 W_{x}^{2} W_{y}^{2}}, \quad B\left(\xi_{1}\right)=\frac{W_{x}^{2}-W_{y}^{2}}{2 W_{x}^{2} W_{y}^{2}}, \\
& A\left(\xi_{2}\right)=\frac{W_{x}^{2} R_{y}^{2}+W_{y}^{2} R_{x}^{2}}{2 W_{x}^{2} W_{y}^{2}}, \quad B\left(\xi_{2}\right)=\frac{W_{x}^{2} R_{y}^{2}-W_{y}^{2} R_{x}^{2}}{2 W_{x}^{2} W_{y}^{2}} .
\end{aligned}
$$

After solving Eqs. (20) and (21) for $W_{x}$ and $W_{y}$, the polarization corrections $\delta \beta_{x}$ and $\delta \beta_{y}$ may be obtained by substituting Eqs. (7), (8) and (11) into Eq. (10).

$$
\begin{aligned}
& \delta \beta_{x}=-\frac{\left(2 \Delta_{2}\right)^{3 / 2}}{2 a_{1} V_{x}^{3} W_{x}^{4}}, \\
& \delta \beta_{y}=-\frac{\left(2 \Delta_{2}\right)^{3 / 2}}{2 b_{1} V_{y}^{3} W_{y}^{4}} .
\end{aligned}
$$

Then the normalized birefringence is given by

$$
B=\frac{\delta \beta_{x}-\delta \beta_{y}}{k}=\frac{2 n_{0} \Delta_{2}^{2}}{V_{y}^{4}}\left[\frac{1}{W_{y}^{4}}-\frac{1}{\left(a_{1} / b_{1}\right)^{4} W_{x}^{4}}\right] .
$$

The modal dispersion is given by

$$
\Delta \tau=\frac{1}{c} \frac{d\left(\delta \beta_{x}-\delta \beta_{y}\right)}{d k}=\frac{2 n_{0} \Delta_{2}^{2}}{c} \frac{d}{d V_{y}}\left\{\frac{1}{V_{y}^{3}}\left[\frac{1}{W_{y}^{4}}-\frac{1}{\left(a_{1} / b_{1}\right)^{4} W_{x}^{4}}\right]\right\} .
$$

The normalized intensity is given by

$$
\begin{aligned}
& S=\frac{\Psi^{2}}{\int_{-\infty}^{\infty} \int_{-\infty}^{\infty} \Psi^{2} d X d Y}, \\
& S=A_{\text {core }} S=\frac{\exp \left[-\frac{1}{2}\left(\frac{X^{2}}{W_{x}^{2}}+\frac{Y^{2}}{W_{y}^{2}}\right)\right]}{W_{x} W_{y}},
\end{aligned}
$$

with $A_{\text {core }}=\pi a_{1} b_{1}$ being the elliptical core area.

\section{Results and Discussion}

\section{A. Spot Size and Intensity Distribution}

Figures 2(a)and (b) show the spot sizes $W_{x}$ and $W_{y}$ as functions of $V_{y}$ for single-clad and double-clad fibers with $a_{1} / b_{1}=1.5$ and 3.0. The results for the singleclad fibers are the same as the theoretical results obtained previously, ${ }^{16}$ and agree well with experimental results. ${ }^{22}$ When the fiber is given a second cladding, the spot size becomes smaller. This means that the intensity distribution is more confined to the fiber center when a depressed inner cladding is introduced (see Fig. 3). Consequently, losses in the claddings may be decreased. This also loosens the angular alignment requirement during splicing.

\section{B. Birefringence}

The case of a single-clad elliptical fiber was analyzed by matching the boundary conditions and by solving the eigenvalue problem with the help of Mathieu functions. ${ }^{2}$ Figure 4 compares the results obtained by such a method with those obtained by our Gaussian approximation. Between $1.3 \leq V_{y} \leq 3$, which is the region of greatest interest when single-mode fibers are 


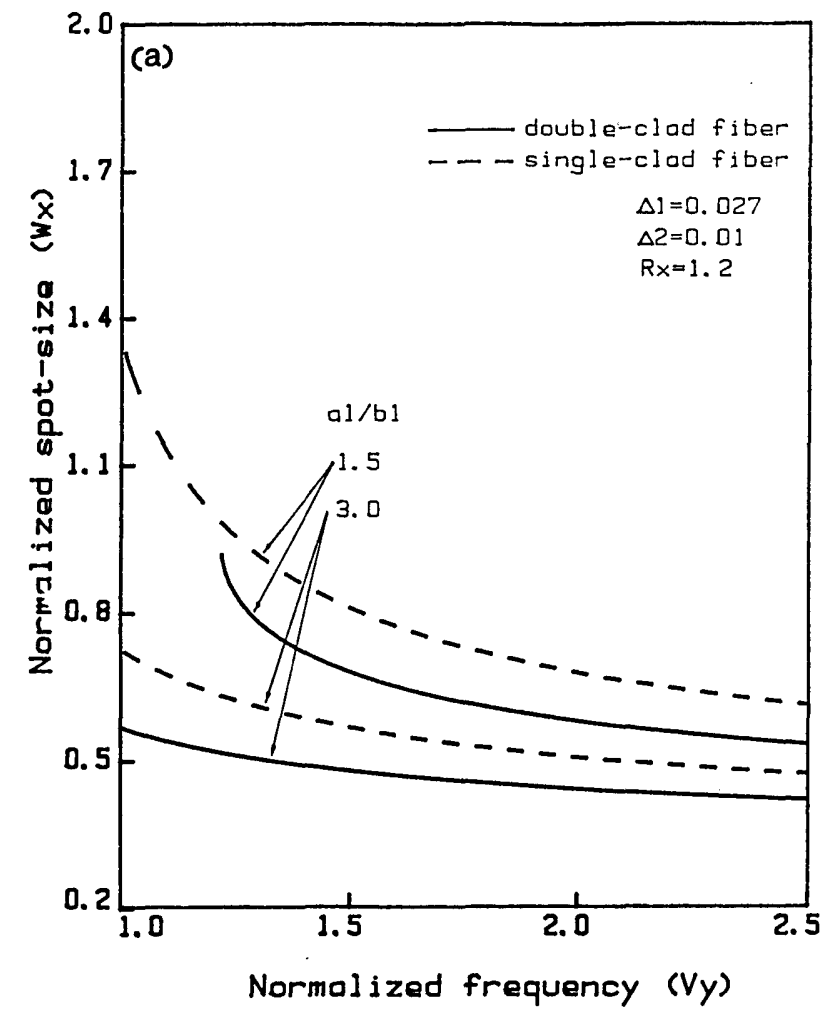

(a)

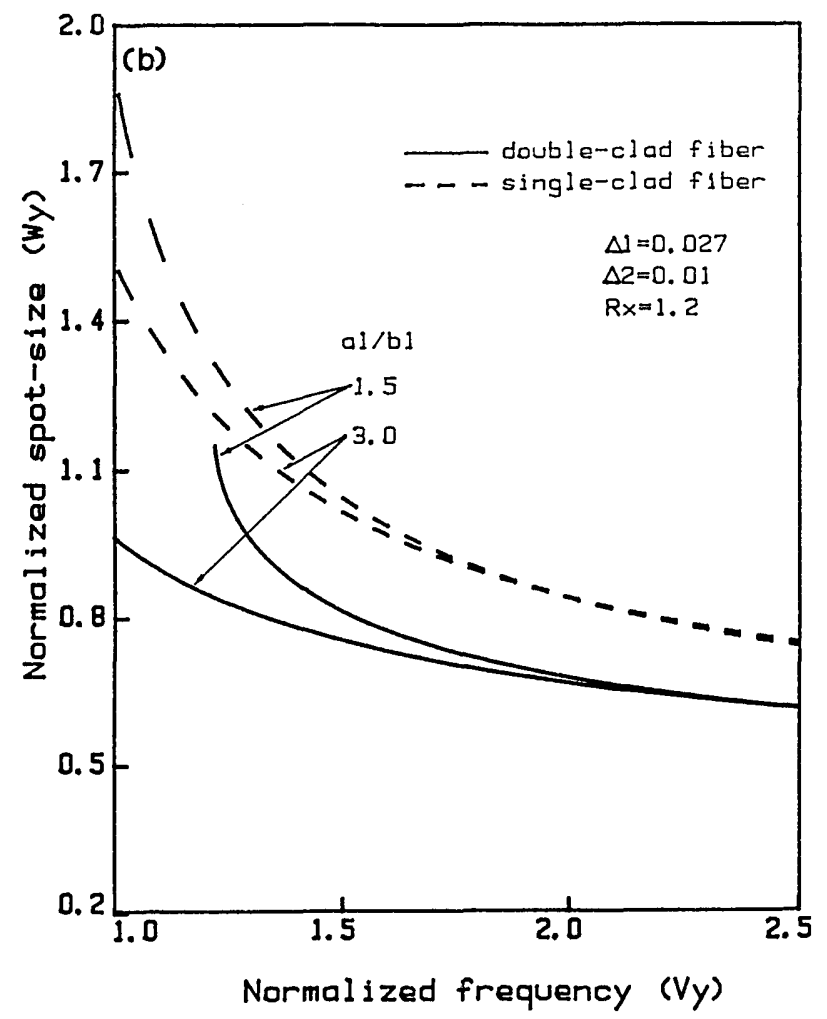

(b)

Fig. 2. (a) Spot size $W_{x}$ vs $V_{y}$ for $a_{1} / b_{1}=1.5$ and 3.0. (b) Spot-size $W_{y}$ vs $V_{y}$ for $a_{1} / b_{1}=1.5$ and 3.0. Solid and dashed lines correspond to double-clad and single-clad elliptical fibers, respectively.

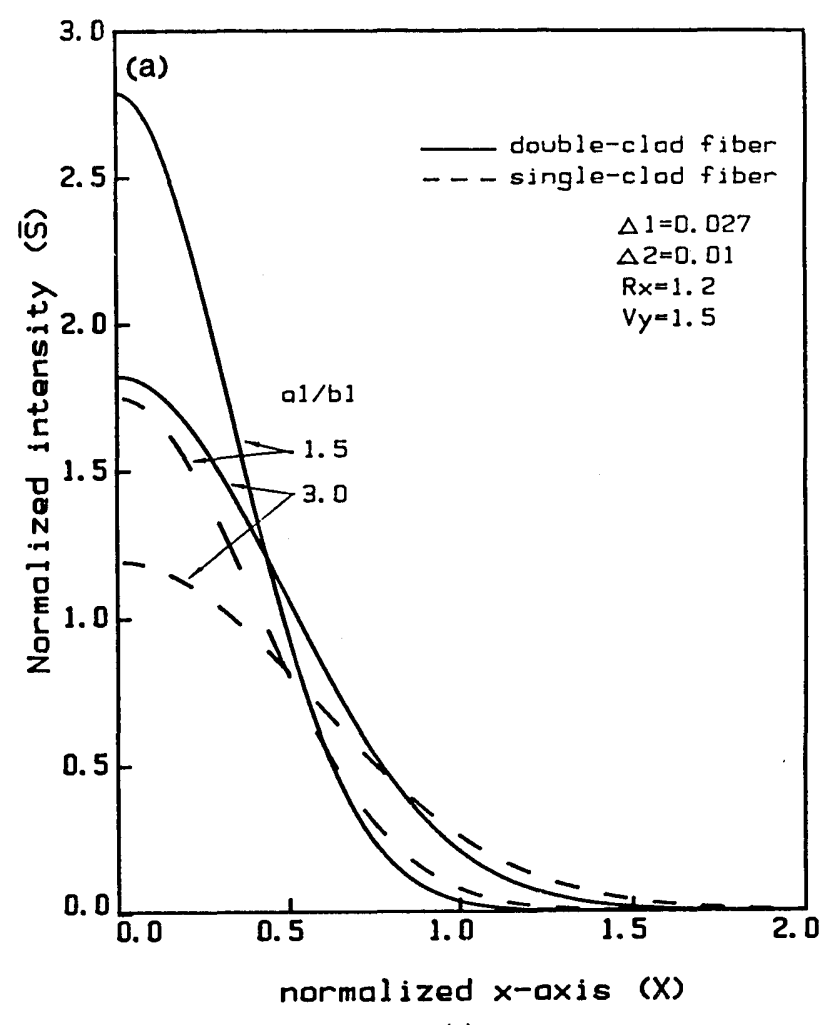

(a)

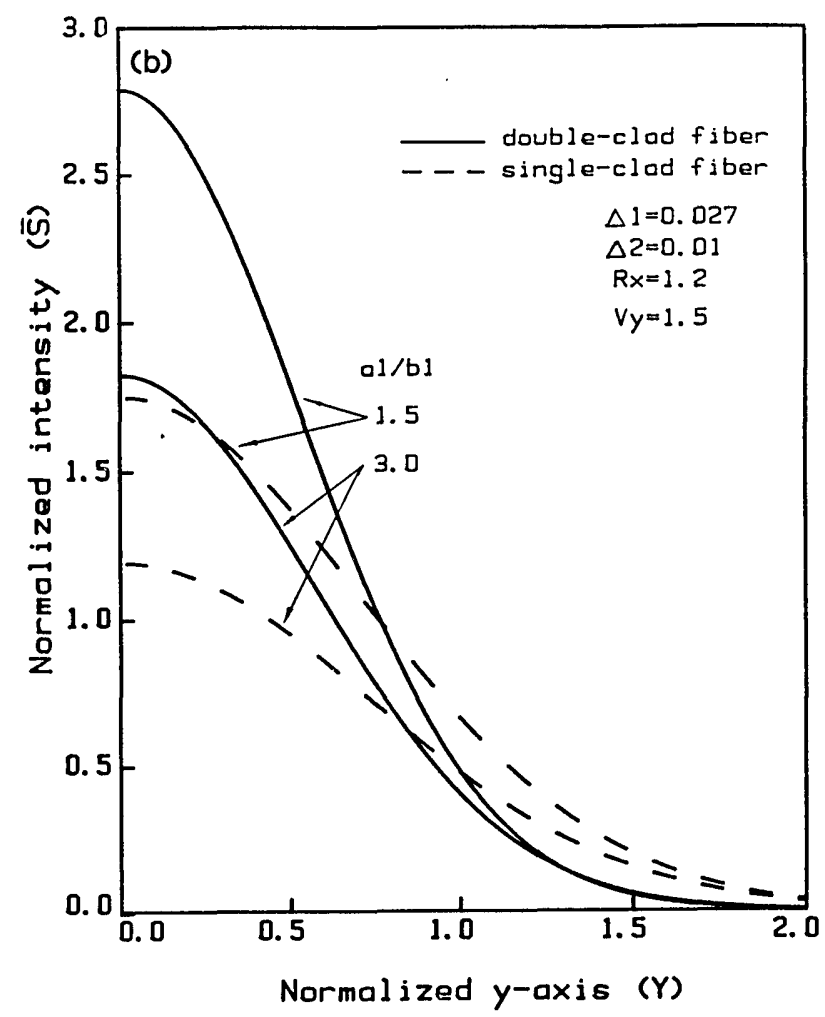

(b)

Fig. 3. (a) Intensity distributions along the minor axis. (b) Intensity distributions along the major axis. Solid and dashed lines correspond to double-clad and single-clad elliptical fibers, respectively. 


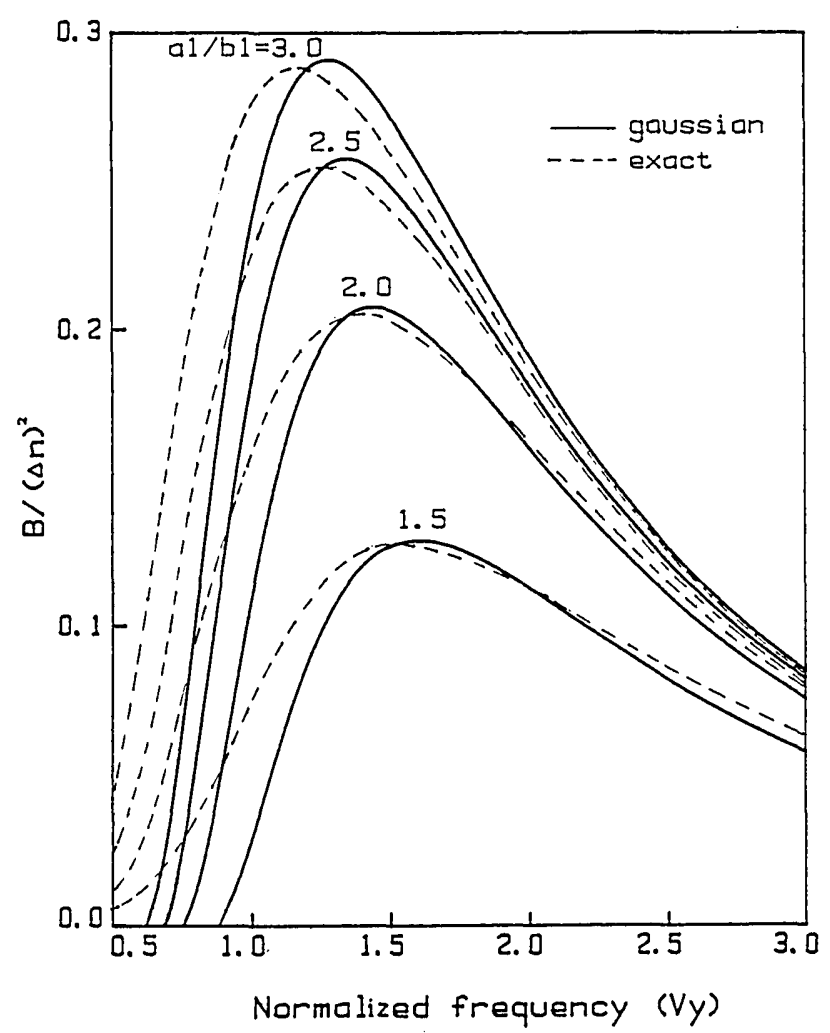

Fig. 4. Comparison between the birefringences obtained by the eigenvalue method using Mathieu's functions and the Gaussian approximation for a single-clad elliptical fiber.

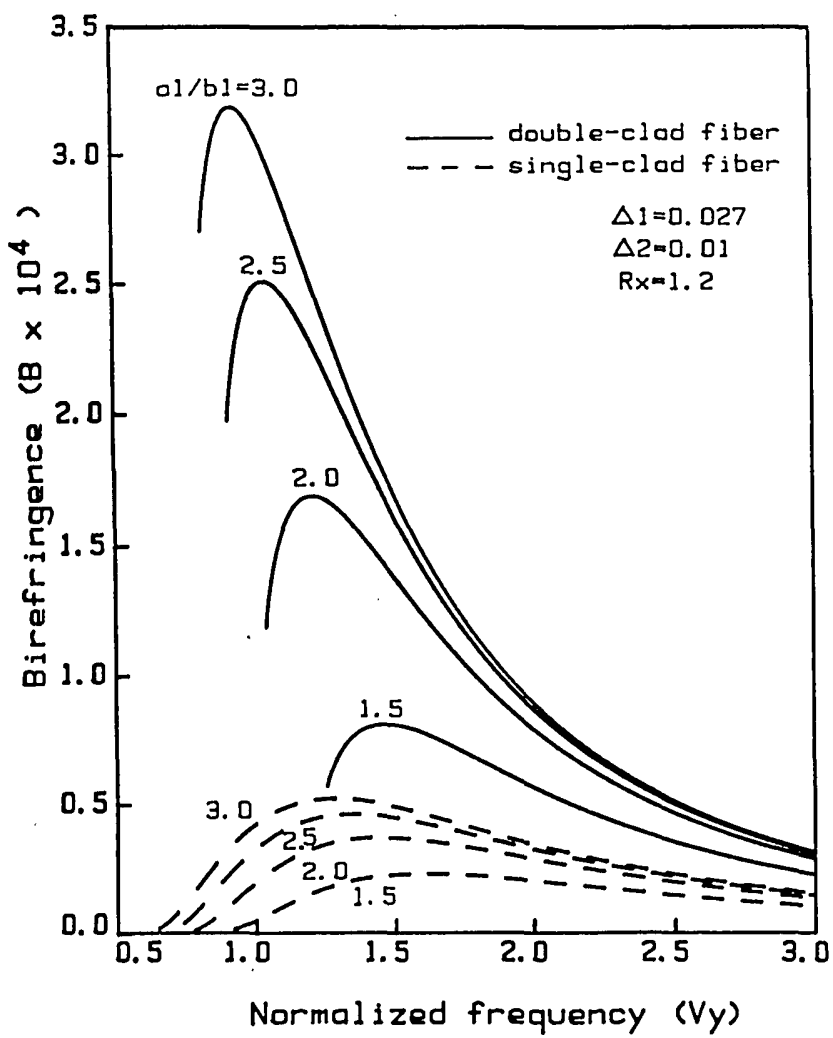

Fig. 5. Normalized birefringence $B$ as a function of $V_{y}$ for $a_{1} / b_{1}=$ $1.5,2.0,2.5$, and 3.0. Solid and dashed lines correspond to doubleclad elliptical fibers and single-clad fibers.

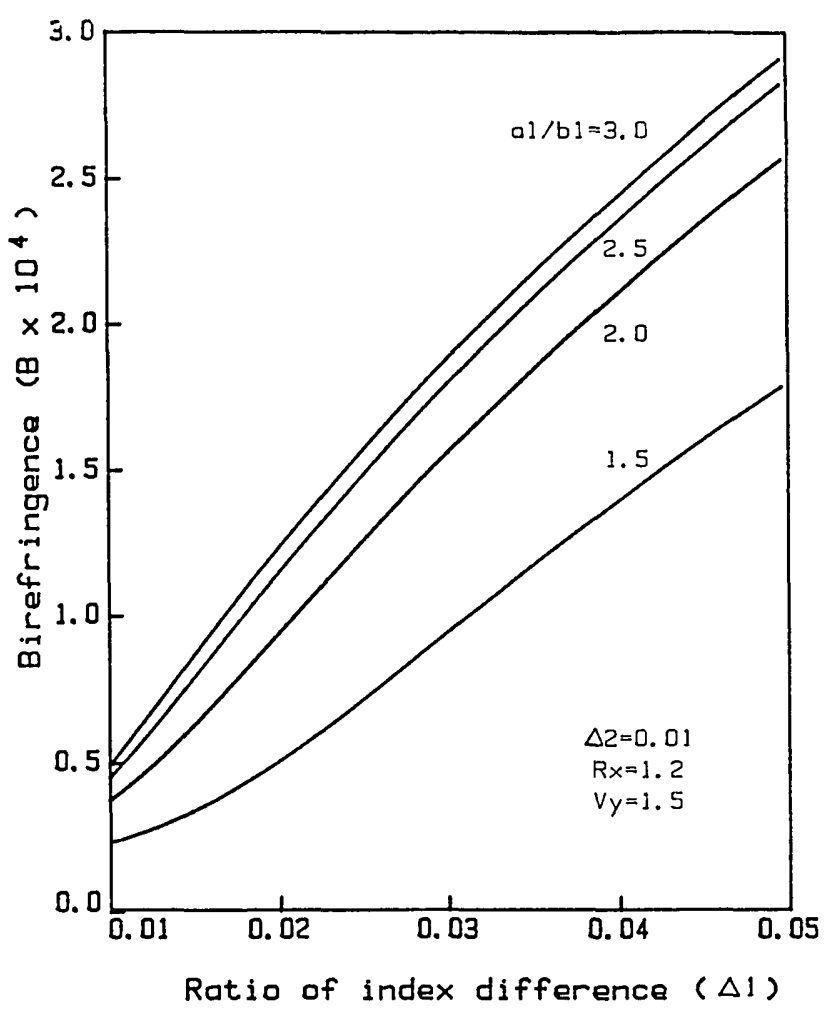

Fig. 6. Normalized birefringence $B$ as a function of $\Delta_{1}$ for $a_{1} / b_{1}=$ $1.5,2.0,2.5$, and 3.0 .

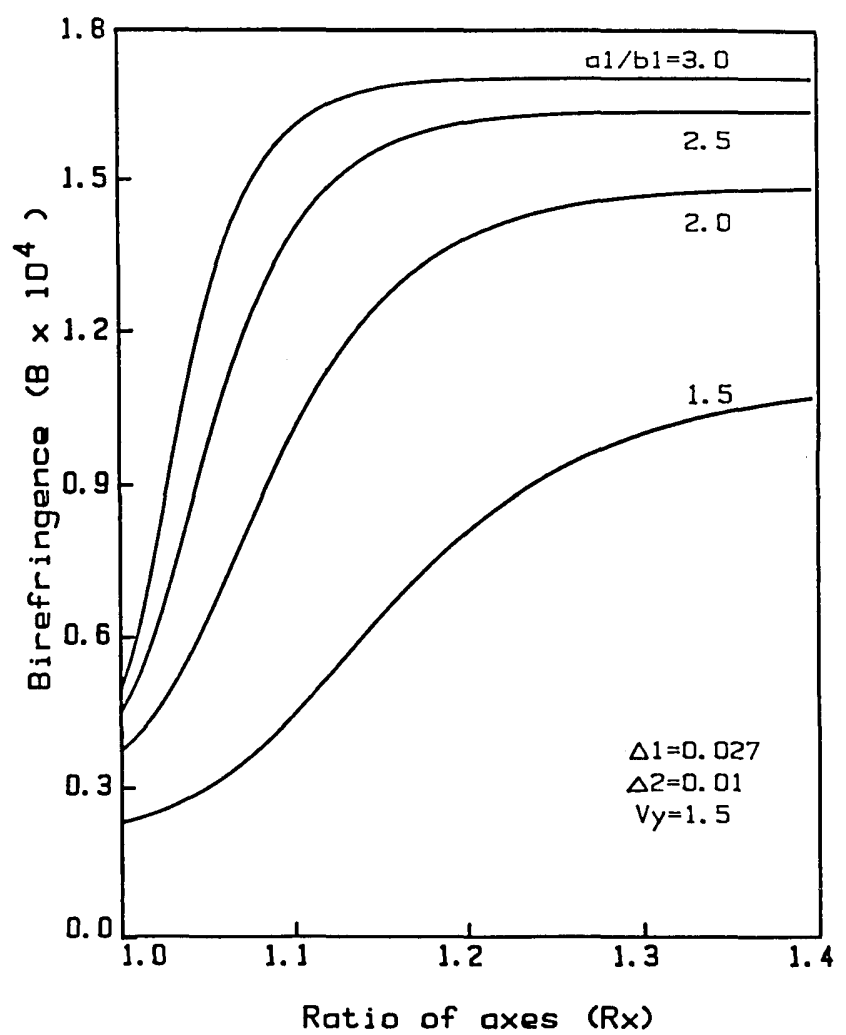

Fig. 7. Normalized birefringence $B$ as a function of $R_{x}$ for $a_{1} / b_{1}=$ $1.5,2.0,2.5$, and 3.0 . 


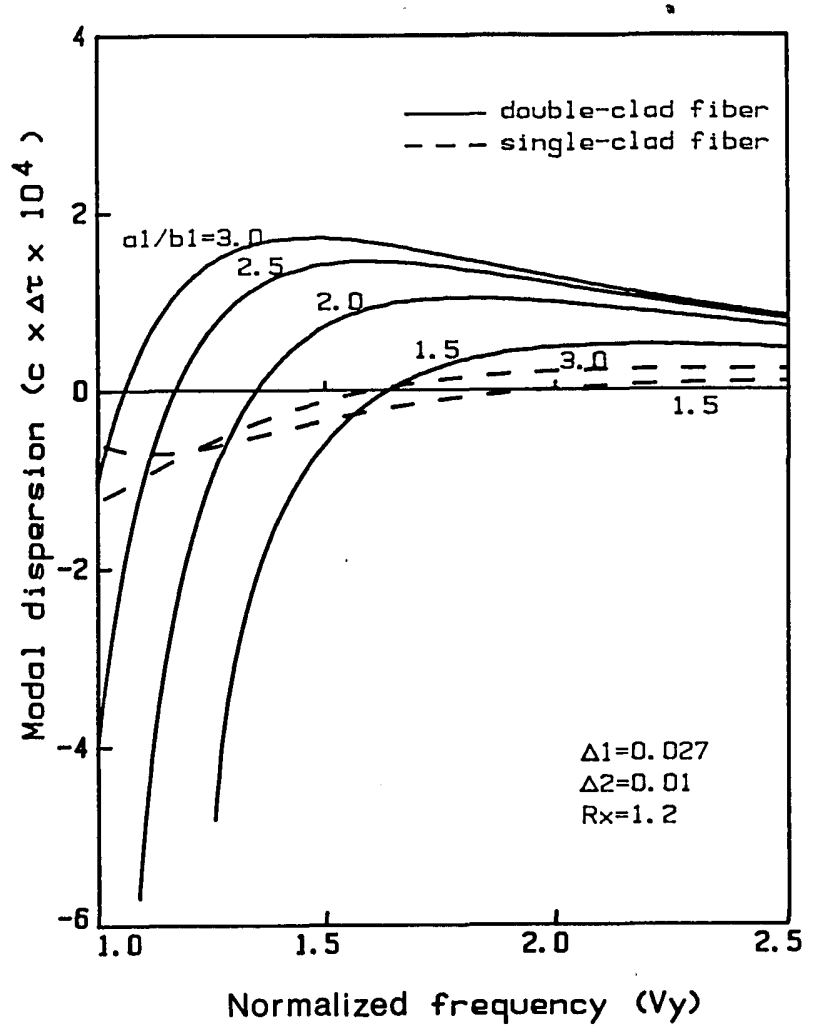

Fig. 8. Modal dispersion as a function of $V_{y}$ for $a_{1} / b_{1}=1.5,2.0,2.5$, and 3.0.

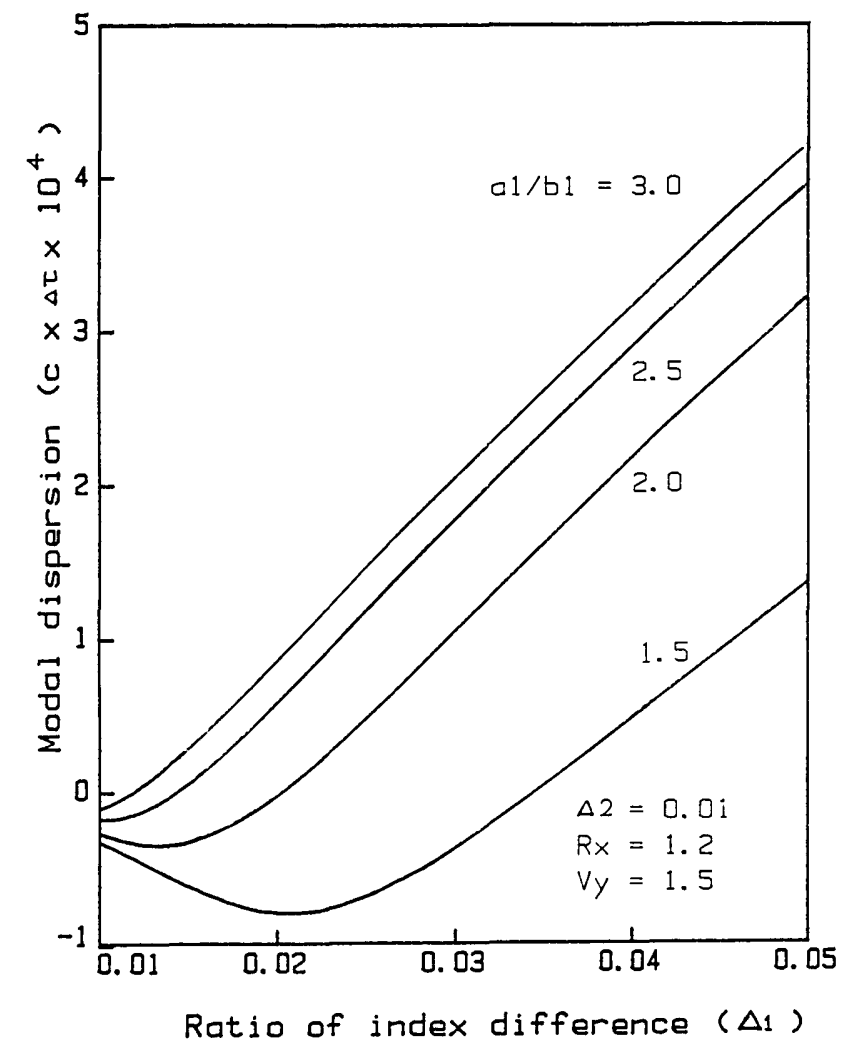

Fig. 9. Modal dispersion as a function of $\Delta_{1}$ for $a_{1} / b_{1}=1.5,2.0,2.5$, and 3.0.

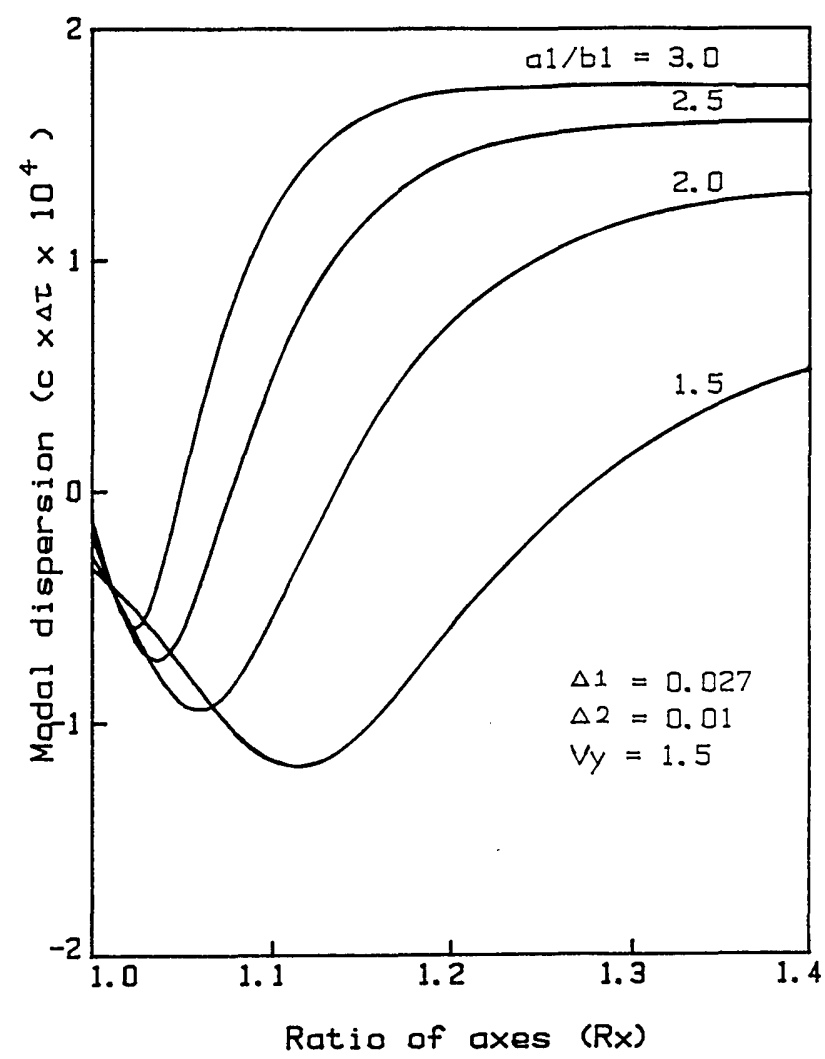

Fig. 10. Modal dispersion as a function of $R_{x}$ for $a_{1} / b_{1}=1.5,2.0,2.5$, and 3.0.

used for information transmission, the two results agree well, with differences $<8 \%$.

Figure 5 shows the normalized birefringence $B$ of DICE fibers as a function of $V_{y}$ for $a_{1} / b_{1}=1.5,2.0,2.5$, and 3.0. The maximum value of $B$ for $a_{1} / b_{1}=3$ for a DICE fiber is $\sim 6 \times$ as large as that for a SCE fiber.

Figure 6 shows $B$ vs $\Delta_{1}$ for $a_{1} / b_{1}=1.5,2.0,2.5$, and 3.0. When $\Delta_{1}=\Delta_{2}=0.01$, the fiber becomes a SCE fiber. The birefringence obviously increases with the refractive index difference $\Delta_{1}$. This allows us to obtain high birefringence in weakly guiding DICE fibers by selecting $\Delta_{1}$; such flexibility is not provided by SCE fibers.

Figure 7 shows $B$ as a function of $R_{x}$, the ratio of the major axis of the core to that of the inner cladding for $a_{1} / b_{1}=1.5,2.0,2.5$, and 3.0. When $R_{x}=1$, the fiber is a SCE fiber; $B$ increases sharply with $R_{x}$ for $R_{x}<1.3$, but changes little beyond $R_{x}=1.4$.

\section{Dispersion}

Figure 8 shows the modal dispersion $c \Delta \tau$ as a function of $V_{y}$ for $a_{1} / b_{1}=1.5,2.0,2.5$, and 3.0. The zero dispersion points of DICE fibers occur at values of $V_{y}$ smaller than those of SCE fibers. The more important fact is that all the cases shown in the figure fall within the single-mode operation range. ${ }^{19}$

Figures 9 and 10 give $c \Delta \tau$ as a function of $\Delta_{1}$ and of $R_{x}$, respectively, for $a_{1} / b_{1}=1.5,2.0,2.5$, and 3.0. Fig- 
ures 8-10 show that the point at which a DICE fiber will have zero dispersion can be significantly shifted by adjusting the ratio $a_{1} / b_{1}$, index difference $\Delta_{1}$, and ratio of axes $R_{x}$. This allows more flexibility in the design of high birefringence and dispersion free fibers.

\section{Conclusion}

By using a scalar variational analysis based on Gaussian approximation of the fundamental mode, we have solved numerically the modal spot size equations for weakly guiding DICE fibers. We have presented the graphic results for the spot size, birefringence, and modal dispersion as functions of various important parameters, such as the normalized frequency $V_{y}$, the difference $\Delta_{1}$ of the refractive index of the core and that of the inner cladding, and the ratio $R_{x}$ of the inner cladding major axis to the core major axis. The field intensity distributions are also given. Moreover, fibers with different ellipticities $a_{1} / b_{1}$ are considered. We have shown that, when compared with a SCE fiber, a DICE fiber can give a smaller spot size, narrower field distribution, higher birefringence, and dispersion-free operation with smaller normalized frequencies.

This research was supported by the Ontario Laser \& Lightwave Research Center and by the National Science \& Engineering Research Council of Canada.

John Lit also works in the Department of Physics \& Computing of Wilfrid Laurier University.

\section{References}

1. C. Yeh, "Elliptical Dielectric Waveguides," J. Appl. Phys. 33, 3235-3243 (1962).

2. R. B. Dyott, J. R. Cozens, and D. G. Morris, "Preservation of Polarization in Optical-Fiber Waveguides With Elliptical Cores," Electron. Lett. 15, 380-382 (1979).

3. S. C. Rashleigh and M. J. Marrone, "Polarization Holding in Elliptical-Core Birefringent Fibers," IEEE J. Quantum Electron. QE-18, 1515-1523 (1982).

4. V. Ramaswamy, W. G. French, and R. D. Standley, "Polarization Characteristics of Noncircular Core Single-Mode Fibers," Appl. Opt. 17, 3014-3017 (1978).

5. K. Kitayama, S. Seikai, N. Uchida, and M. Akiyama, "Polarization-Maintaining Single-Mode Fiber With Azimuthally Inhomogeneous Index Profile," Electron. Lett. 17, 419-420 (1981).

6. T. Okoshi, K. Oyamada, M. Nishimura, and H. Yokota, "SideTunnel Fiber: An Approach to Polarization-Maintaining Optical Wave-Guiding Scheme," Electron. Lett. 18, 824-826 (1982).
7. V. Ramaswamy, R. H. Stolen, M. D. Divine, and W. Plieble, "Birefringence in Elliptically Clad Borosilicate Single-Mode Fibers," Appl. Opt. 18, 4080-4084 (1979).

8. S. C. Rashleigh and M. J. Marrone, "Polarization-Holding in a High-Birefringence Fiber," Electron. Lett. 18, 326-327 (1982).

9. T. Hosaka, Y. Sasaki, J. Noda, and M. Horiguchi, "Low-Loss and Low-Crosstalk Polarization-Maintaining Optical Fibers," Electron. Lett. 21, 920-921 (1985).

10. R. D. Birch, M. P. Varnham, D. N. Payne, and E. J. Tarbox, "Fabrication of Polarization-Maintaining Fibers Using Gas- ' Phase Etching," Electron Lett. 18, 1036-1038 (1982).

11. R. H. Stolen, W. Pleibel, and J. R. Simpson, "High-Birefringence Optical Fibers by Preform Deformation," IEEE/OSA J. Lightwave Technol. LT-2, 639-641 (1984).

12. J. Noda, K. Okamoto, and Y. Sasaki, "Polarization-Maintaining Fibers and Their Applications," IEEE/OSA J. Lightwave Technol. LT-4, 1071-1089 (1986).

13. S. C. Rashleigh and M. J. Marrone, "Temperature Dependence of Stress Birefringence in an Elliptically Clad Fiber," Opt. Lett. 8, 127-129 (1983).

14. A. N. Chester, S. Martellucci, and A. M. Verga Scheggi, "Optical Fiber Sensors," NATO ASI Series E: Appl. Sci. 132, 18-19 (1987).

15. A. Kumar, R. K. Varshney, and K. Thyagarajan, "Birefringence Calculations in Elliptical-Core Optical Fibers," Electron. Lett. 20, 112-113 (1984).

16. S. Sarkar, K. Thyagarajan, and A. Kumar, "Gaussian Approximation of the Fundamental Mode in Single Mode Elliptic Core Fibers," Opt. Commun. 49, 178-183 (1984).

17. R. B. Dyott and J. Bello, "Self-Locating Elliptically Cored Fiber with an Accessible Guiding Region," Electron. Lett. 18,980-981 (1982).

18. R. K. Varshney, R. Srivastava, and R. V. Ramaswamy, "Characterization of Highly Elliptical Submicron Core Polarization Preserving Fibers: Theory and Experiment," Appl. Opt. 27, 31143120 (1988).

19. R. K. Varshney and A. Kumar, "Effect of Depressed Inner Cladding on the Polarization Characteristics of Elliptical-Core Fibers," Opt. Lett. 9, 522-525 (1984).

20. A. W. Snyder and J. D. Love, Optical Waveguide Theory (Chapman \& Hall, London, 1983).

21. More than eight definitions have been proposed for spot size, and no agreement has been reached for a universally accepted one [E.-G. Neumann, Single-Mode Fibers (Springer-Verlag, New York, 1988), pp. 221-229.] The definition we used here is essentially that proposed by Snyder, "Understanding Monomode Optical Fibers," Proc. IEEE 69, 6-12 (1981).

22. R. Yamauchi, T. Murayama, Y. Kikuchi, Y. Sugawara, and K. Inada, "Spot Sizes of Single-Mode Fibers with a Non-Circular Core," in Technical Digest, Fourth International Conference on Integrated Optics and Optical Fiber Communication, Tokyo (June 1983), 40-41. 